\title{
Multidimensional simulations of pair-instability supernovae
}

\author{
A. A. Baranov ${ }^{1}$, P. Chardonnet ${ }^{1}$, V. M. Chechetkin ${ }^{2,3}$, A. A. Filina ${ }^{1}$, and M. V. Popov ${ }^{1,2}$ \\ 1 LAPTh, Univ. de Savoie, CNRS, BP 110, 74941 Annecy-le-Vieux, France \\ e-mail: chardonnet@lapp.in2p3.fr \\ 2 Keldysh Institute of Applied Mathematics, Russian Academy of Science, Miusskaya sq. 4, 125047 Moscow, Russia \\ ${ }^{3}$ National Research Nuclear University "MEPhI", Kashirskoe sh. 31, 115409 Moscow, Russia
}

Received 18 February 2013 / Accepted 9 May 2013

\begin{abstract}
According to theoretical models, massive stars with masses within the 100-250 $M_{\odot}$ range should explode as pair-instability supernovae (PISNe). Since the first stars of the Universe are believed to be very massive, these supernovae should play a significant role in the early stages of its history. But these stars represent the last unobserved population, owing to detection limits of current telescopes. In this work we analyze pair-instability supernovae explosions using various numerical codes. We evolve series of the configurations of oxygen cores to establish a range of masses and initial conditions where this type of explosion is possible. We also study the role of possible instabilities in the propagation of shockwaves during the last stage of the explosion. This investigation could help us to predict the observational properties of PISNe for future space and ground telescopes.
\end{abstract}

Key words. stars: Population III - supernovae: general - hydrodynamics - instabilities

\section{Introduction}

The first stars of the Universe, called Population III stars (Pop III), are rapidly becoming an important subject of investigation from the point of view of theory and observations. The formation of these stars hundreds of millions of years after the Big Bang marks the end of what is called the "Dark Age". Today's telescopes cannot look far enough into the cosmic past, so we do not have direct observations of how the primordial stars were formed. This new window is paramount for astrophysics and cosmology. Population III stars are formed with primordial nucleosynthesis elements and are responsible for the formation of the first metals in the Universe. The change in chemical composition also affects the initial mass function of stars. The energy scattered in the newborn Universe will drastically influence its history. Certainly, the new generation of instruments will give us an opportunity to test theoretical ideas about the formation of the first stars.

To study these stars, we need sophisticated numerical simulations (Bromm et al. 2003; Bromm \& Larson 2004). Pop III stars also have the potential to produce gamma-ray bursts (GRBs), which may provide one of the most promising methods of directly probing the final stages of Pop III stars (Chardonnet et al. 2010; de Souza et al. 2011).

Among these first-generation stars, an important role was played by massive stars. The Jean's mass favors the creation of very massive objects during star formation by condensing a nuclear cloud. Numerical simulations predict that Pop III stars could have masses as high as a few hundred solar masses. As these stars evolve, physical conditions in the center lead to the development of specific type of hydrostatic instability through electron-positron pairs creation (pair instability).

As shown by many numerical simulations (Barkat et al. 1967; Woosley et al. 2002; Waldman 2008), these very massive stars could end their life, depending on mass, either by producing pair-instability supernovae (PISNe), leaving no remnant, or by collapse to a black hole. In the case of PISNe, the energy release is tremendous and could possibly be seen with new telescopes (James Webb Space Telescope, European Extremely Large Telescope).

In this work we analyze the PISN explosion again. We present the results of one-dimensional simulations and analysis of the fate of a star depending on physical conditions. Many recent articles (Chen et al. 2011; Joggerst et al. 2011) address the problem of multidimensional simulations of a PISN explosion. Such studies are needed to compute light curves and time of appearance of lines of chemical elements, so they allow us to predict the observational signatures of these supernovae. We present 2D simulations of PISN explosion based on the idea of nonuniform explosion and compare the results with the case of uniform explosion in stellar core.

\section{Pair-instability supernovae}

One of the first works that has considered electron-positron pairs creation inside stars and predicted instability caused by this process was the paper of Fowler \& Hoyle (1964). The density-temperature relations for massive stars $\left(30 M_{\odot}\right.$ and higher) were computed, using a polytropic structure approximation and the assumption that a star evolves through series of quasi-equilibrium states. A variable number of electron-positron pairs was taken into account by the authors. They discovered that when the central temperature of a star reaches values $\sim 2 \times 10^{9} \mathrm{~K}$ intensive creation of electron-positron pairs occurs. It increases the energy losses by neutrino through a pair annihilation process, $\mathrm{e}^{+}+\mathrm{e}^{-} \rightarrow v_{\mathrm{e}}+v_{\mathrm{e}}$, and accelerates the contraction, raising central temperature. The rise of temperature creates new pairs, and at some point, energy supply from the contraction becomes insufficient to maintain production of additional pairs. As a result implosion of the core develops until the temperature reaches values necessary for explosive nuclear burning.

Instability caused by pair production could also be described in terms of thermodynamics. Under the assumption of 
an adiabatic process, the electron-positron pairs creation reduces adiabatic index $\Gamma=(\partial \ln p / \partial \ln \rho)_{S}$ to values less than $4 / 3$. If a significant part of a star reaches conditions where $\Gamma \leq 4 / 3$, the star becomes very "soft" and dynamically unstable.

First dynamical calculations of a star that undergoes pair instability were performed by Barkat et al. (1967). It was shown in this paper that implosion of $40 M_{\odot}$ oxygen star could be reversed into explosion by nuclear burning in the center. Nuclear energy release was $\sim 6 \times 10^{51} \mathrm{erg}$, corresponding to consumption of several solar masses of oxygen. It was enough not only to reverse the collapse but also to disrupt the star totally without any remnant. Further investigation of the evolution of very massive stars is found in numerous works (Rakavy et al. 1967; Rakavy \& Shaviv 1967, 1968). It was concluded that stars heavier than $25 M_{\odot}$ are pair-unstable. The cores lighter than this limit pass pair-instability zone and evolve up to the Fe-He transition region. Dynamical calculations of unstable oxygen cores for 45-60 $M_{\odot}$ were performed by Fraley (1968), who obtained an oscillation for the lightest core and explosion for the heavier cores from this interval. First detailed evolutional calculations of helium cores were performed by Arnett (1972). He has demonstrated that the cores consist mainly of oxygen when reaching the pair-instability zone. Dynamical calculations of helium cores with masses $64 M_{\odot}$ and $100 M_{\odot}$ were presented by Arnett $(1973,1974)$. The cores exploded and the lighter one left a $2.2 M_{\odot}$ silicon remnant. Wheeler (1977) found that the cores with masses $10^{3}-10^{4} M_{\odot}$ collapse.

These numerical simulations have shown that the fate of a star depends on its mass and its configuration. Apparently there is a window of masses when a star explodes as pair-instability supernova (Woosley et al. 2002). If the star is too light it avoids pair instability and could collapse to a black hole or neutron star owing to dissociation of iron at higher temperatures. If the star is too heavy, nuclear burning is not enough to reverse the implosion caused by pair creation. In the middle mass range, there is a possibility of explosion. A detailed scenario of the dynamical stage depends on the balance between the rate of neutrino losses, speed of contraction, and rate of nuclear energy release.

\section{Numerical approach}

To investigate the behavior of pair-unstable stars, we performed various hydrodynamical simulations. With the one-dimensional (1D) Lagrangian code, we studied the fate of oxygen cores depending on mass and initial configuration. Application of 1D Lagrangian schemes (not only for evolutionary, but also for dynamical calculations) is common in modern research (Woosley et al. 2007; Waldman 2008; Kasen et al. 2011) since it has some advantages in solving the system of equations including nuclear burning.

To study the last stage of explosion when shockwave propagates outward, we applied a two-dimensional (2D) code. There are a few recent modelizations of PISNe in 2D (Chen et al. 2011; Joggerst et al. 2011). In both cases a modern astrophysical code, CASTRO, has been used. This code is based on a high-order radiation-hydrodynamics solver (Almgren et al. 2010), includes self-gravity, and allows following nucleosynthesis and energy output from nuclear burning during the explosion. But to investigate the influence of hydrodynamical solvers we applied our own numerical code based on the piecewise parabolic method on a local stencil (PPML; Popov \& Ustyugov 2007; Popov 2012).

Of course a real star is three-dimensional, so detailed and precise description requires consideration of all physical processes inside a star in 3D. Extension of PPML code to full
Table 1. Presupernova models and parameters of explosion.

\begin{tabular}{lccccc}
\hline \hline $\begin{array}{l}M_{\mathrm{c}} \\
{\left[M_{\odot}\right]}\end{array}$ & $\begin{array}{c}E_{\mathrm{bind}} \\
{\left[10^{51} \mathrm{erg}\right]}\end{array}$ & $\begin{array}{c}\rho_{\mathrm{c}} \\
{\left[10^{5} \mathrm{~g} / \mathrm{cc}\right]}\end{array}$ & $\begin{array}{c}T_{\max } \\
{\left[10^{9} \mathrm{~K}\right]}\end{array}$ & $\begin{array}{c}E_{\text {nuc }} \\
{\left[10^{51} \mathrm{erg}\right]}\end{array}$ & Fate \\
\hline 60 & -5.54 & 0.87 & 4.07 & 20.9 & PISN \\
60 & -5.48 & 1.15 & 4.07 & 21.0 & PISN \\
70 & -6.10 & 1.0 & - & - & $\mathrm{CC}$ \\
70 & -5.78 & 1.3 & - & - & $\mathrm{CC}$ \\
70 & -5.27 & 1.7 & 4.52 & 29.4 & PISN \\
70 & -4.87 & 2.0 & 4.16 & 25.9 & PISN \\
70 & -3.67 & 3.0 & 3.74 & 18.8 & PISN \\
78 & -4.59 & 2.0 & - & - & CC \\
78 & -3.73 & 2.5 & 4.18 & 28.8 & PISN \\
78 & -2.96 & 3.0 & 3.84 & 23.1 & PISN \\
90 & -5.19 & 1.5 & - & - & CC \\
90 & -3.78 & 2.0 & - & - & CC \\
90 & -3.01 & 2.3 & - & - & CC \\
90 & -2.07 & 2.7 & 4.77 & 38.7 & PISN \\
90 & -1.85 & 2.8 & 4.30 & 34.9 & PISN \\
90 & -1.43 & 3.0 & 4.11 & 38.7 & PISN \\
95 & -1.87 & 1.5 & - & - & CC \\
95 & -1.11 & 2.6 & 4.39 & 39.9 & PISN \\
95 & -0.98 & 2.8 & 4.31 & 37.1 & PISN \\
95 & -0.63 & 3.0 & 4.20 & 35.0 & PISN \\
100 & -2.43 & 2.0 & - & - & CC \\
100 & -1.12 & 2.5 & 4.56 & 44.7 & PISN \\
100 & -0.69 & 2.65 & 4.32 & 41.5 & PISN \\
\hline & & & & & \\
\hline
\end{tabular}

Notes. $M_{\mathrm{c}}$ is the mass of the oxygen core, $E_{\text {bind }}$ the initial binding energy, $\rho_{\mathrm{c}}$ the initial central density, $T_{\max }$ the maximum temperature at the center at the moment of reversal of collapse, $E_{\text {nuc }}$ the total nuclear energy release. PISN - the model explodes without any remnant, $\mathrm{CC}-$ collapse at the center of the core.

3D case will be made in future. In the current research we focus only on the hydrodynamics of explosion applying new highquality hydrodynamic PPML solver.

\subsection{Modelling in $1 D$}

We considered dynamics of massive stellar cores that are unstable due to pair creation. One of the key questions of supernova modeling is the choice of the initial configuration. A very common approach is to compute the presupernova configuration by evolutionary codes. But evolutionary codes usually assume spherical symmetry, and any asymmetric process, such as convection or rotation, can only be taken into account in some effective way with approximation. Also detailed computation of stellar evolution requires considering many physical processes that have uncertain descriptions. This lead to precise presupernova configurations possibly being unknown.

Instead of taking initial conditions from evolutionary calculations, we used a polytropic structure approximation with index $4 / 3$. For each core having mass $M_{\mathrm{c}}$, we built several configurations by choosing different values of central density, $\rho_{\mathrm{c}}$. This allowed us to consider models with different values of binding energy, $E_{\text {bind }}$, for each $M_{\mathrm{c}}$ (see Table 1$)$. Although this approach simplifies description of the stellar structure, it gives a possibility to explore a wider variety explosion scenarios that depend on initial configuration. Thermodynamical quantities at the center that we have chosen and values of binding energy are very close to the results of evolutionary calculations (Waldman 2008).

The question of the presence of massive hydrogen envelope in very massive stars is under debate. It is not clear that such stars keep this envelope up to the moment of explosion since it is 


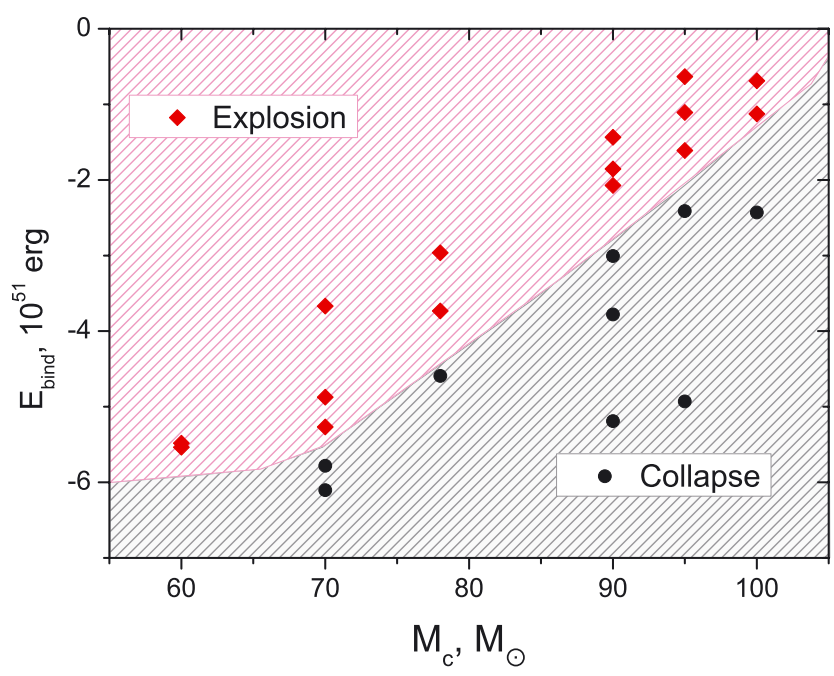

Fig. 1. Fate of a star depending on its mass, $M_{\mathrm{c}}$, and binding energy, $E_{\text {bind. }}$ Explosion is marked by diamonds and collapse is marked by circles.

very weakly bound, for example, it was shown by Woosley et al. (2007) that the envelope could be expelled in previous stages of evolution. In some works (e.g. Nozawa et al. 2003) the dynamics of very massive stars was considered by taking the massive envelope into account. In others (e.g. Kasen et al. 2011), only the cores were considered. In this work we only followed the dynamics of the cores without an envelope.

For the 1D computations we developed a numerical code based on the standard Lagrangian approach (e.g. Dunina-Barkovskaya \& Imshennik 2003). The equation of state that we used (Blinnikov et al. 1996) takes the birth of electronpositron pairs into account. Energy release from nuclear burning and neutrino losses were taken into account. Nuclear burning was followed by $\alpha$-chain of reactions up to ${ }^{56} \mathrm{Ni}$.

Summarized results of our computations are presented in Table 1. The first important fact is that for each core mass, $M_{\mathrm{c}}$, there is some critical value $E_{\text {bind }}^{*}$ of initial binding energy that determines the fate of the core: explosion (PISN) if $E_{\text {bind }}>E_{\text {bind }}^{*}$ or core-collapse ${ }^{1}(\mathrm{CC})$ for $E_{\text {bind }}<E_{\text {bind }}^{*}$. Two regions could be seen clearly in the $M_{\mathrm{c}}-E_{\text {bind }}$ diagram (Fig. 1). This behavior could be explained by the fact that models with lower $E_{\text {bind }}$ (higher absolute value of $E_{\text {bind }}$ ) gain higher kinetic energy to the moment of oxygen ignition and proceeds faster to $\mathrm{Fe}-\mathrm{He}$ transition zone (photodissociation). Thus outer layers of the core do not have enough time to bounce and expand. Therefore the pressure on the central part could not be reduced. Photodissociation dramatically drops the pressure in the center, and the core collapses. The critical value $E_{\text {bind }}^{*}$ tends to zero with a growth of $M_{\mathrm{c}}$. Considering that for a stable nonrotating configuration the binding energy should be negative, we can propose the mass limit for the explosion of nonrotating oxygen core at a value of about $110 M_{\odot}$. This value is in good agreement with results of the previous works (Ober et al. 1983; Bond et al. 1984). Rotation could push the mass limit of explosion to higher values (Glatzel et al. 1985).

An interesting correlation has been found for the models that explode: value of total nuclear energy release, $E_{\text {nuc }}$, increases with maximum temperature $T_{\max }$ reached at the center (Fig. 2). This dependence could be described in a similar way

\footnotetext{
1 Collapse of the central part of the core with possible ejection of outer layers.
}

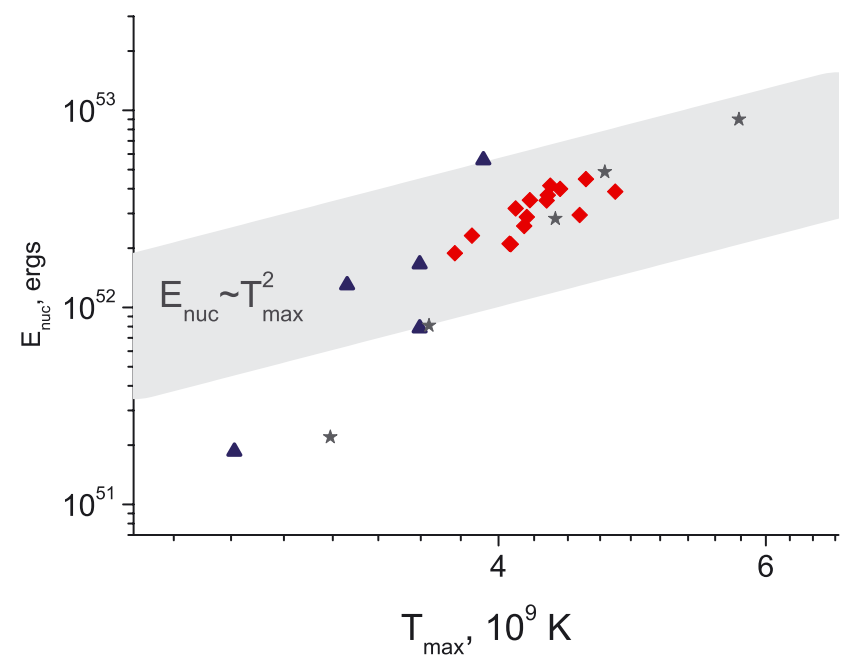

Fig. 2. Nuclear energy release as a function of maximum temperature (diamonds). The slope $E \propto T^{2}$ is shown. For comparison data from Arnett (1996) (stars) and Ober et al. (1983) (triangles) are shown.

to Ayasli \& Joss (1982). For the case of thermonuclear flash in neutron star they have shown that the mass of consumed nuclear fuel is proportional to the square of burning temperature.

\subsection{Numerical explosion in multi-D}

To study the role of hydrodynamical instabilities on process of explosion we have performed 2D computations and compared them with recent works (Chen et al. 2011; Joggerst et al. 2011).

Hydrodynamic simulations were performed with a numerical code based on the PPML (Popov \& Ustyugov 2007; Popov 2012). The key PPML-procedure written on FORTRAN could be found in Ustyugov et al. (2009). This method is an improvement over the popular piecewise parabolic method (PPM) suggested by Colella et al. (1984) for compressible flows with strong shocks and extended by Dai et al. (1994) for magnetohydrodynamics. The PPML method has demonstrated high accuracy in both smooth and discontinued solutions. It could be more suitable for problems where the requirement of low dissipation is crucial. For example, PPML was successfully implemented for direct turbulence simulation (Kritsuk et al. 2009). In our case low dissipation of PPML is needed to describe shocks and contact discontinuities with higher accuracy.

For current study we chose a simplified physical model of explosion. We neglected the energy feedback from nuclear reactions and gravity changes in the source of explosion, trying to obtain the principal possibility of a total disruption of the stellar core to many fragments in the case of a very massive progenitor.

With our hydrodynamic SN model we investigated a Pop III star with a $100 M_{\odot}$ oxygen core assuming rotational symmetry. The oxygen core has the radius $r=0.29 R_{\odot}$, beyond which a uniform density of $\sim 1 \mathrm{~g} / \mathrm{cm}^{3}$ was set for the uniform atmosphere. We used a polytropic model of a star with index $\gamma=4 / 3$. The initial configuration was calculated from the condition of hydrostatic equilibrium of a nonrotating ideal gas sphere with a polytropic equation of state (EOS) $P=K \rho^{\gamma}$, where $K=8.225 \times 10^{15}$, $P$, and $\rho$ are expressed in CGS units. Temperature was computed as $P=\rho R T / \mu$, where $R$ is the universal gas constant and $\mu$ the mean molecular weight, which was assumed to be $\mu=0.37143$. This value of $\mu$ gives the ideal gas approximation, which is very close to the full realistic EOS in the considered range of density and temperature. 

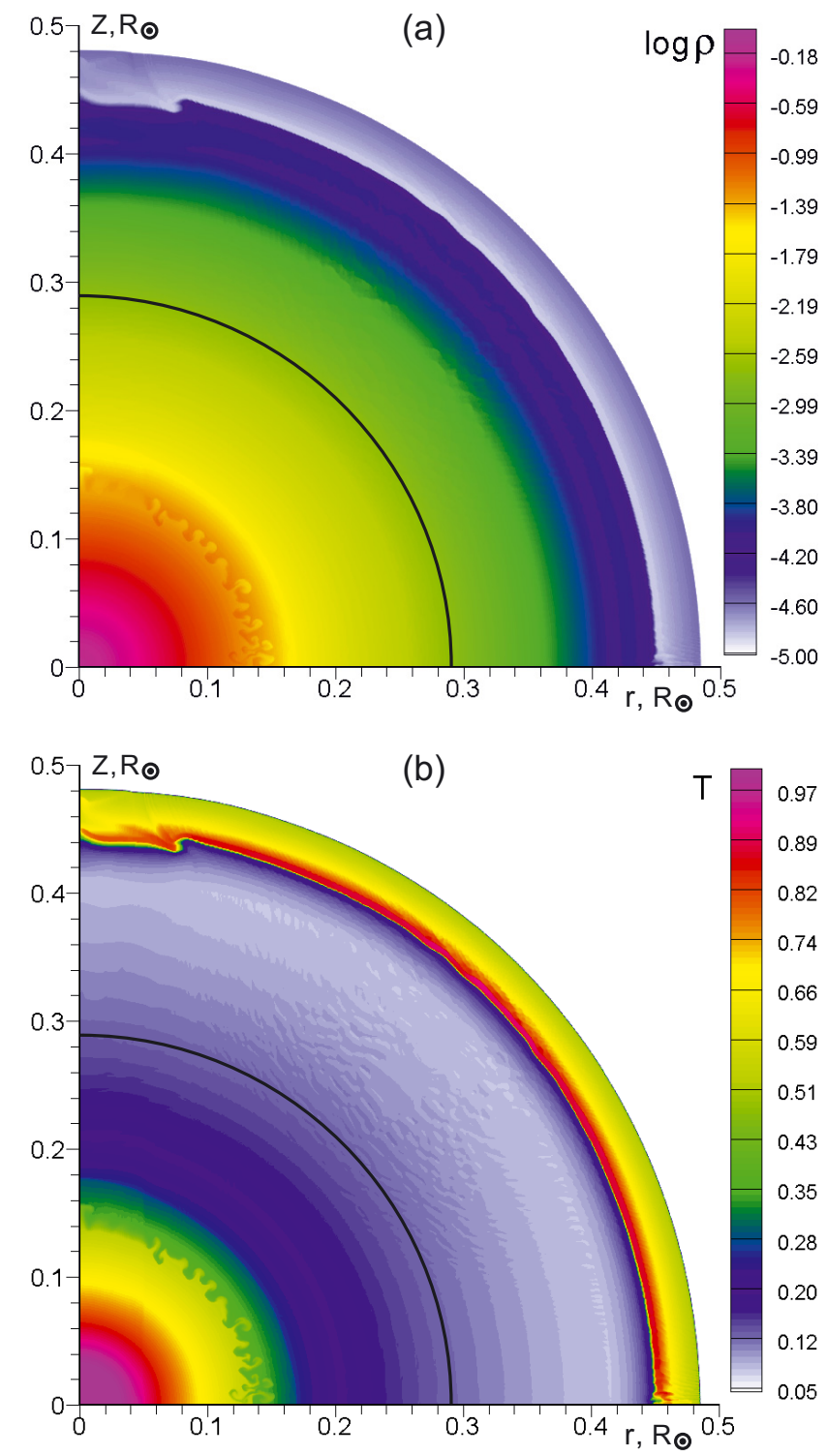

Fig. 3. SN model with central ignition for $t=28 \mathrm{~s}$. Logarithm of density a) is shown in units of $\rho_{\mathrm{c}}=2.65 \times 10^{5} \mathrm{~g} / \mathrm{cm}^{3}$. Temperature b) is shown in units of $T_{\mathrm{c}}=2.36 \times 10^{9} \mathrm{~K}$.

We simulated the explosion by depositing the thermal energy in the central region in the two variants. In the first case the energy $E=5 \times 10^{52}$ erg was uniformly deposited within the radius $r_{\mathrm{e}}=0.1 R_{\odot}$, which contains $60 M_{\odot}$. In the second variant the energy was inserted by the series of ten ignition bubbles at $t=0 \mathrm{~s}$. All of the bubbles had different energy values and sizes distributed in a stochastic way. The bubbles were randomly put within $0.06 R_{\odot}$ of the center. The total energy deposited was also $E=5 \times 10^{52} \mathrm{erg}$. This nonuniformity could present some inhomogeneities in the core that occur prior to explosion.

Nuclear burning in the center of a star could cause the development large-scale convection (Arnett \& Meakin 2011). If convection occurs prior to the moment of pair instability, the contraction and explosion could be nonsymmetrical. Inhomogeneities in temperature and density could cause ignition spots to occur in the core.

The results of computing of the uniform explosion are presented in Fig. 3. It shows density on a logarithmic scale and temperature for the moment $t=28 \mathrm{~s}$. The solid line represents the initial position of the boundary of the core. The shock,
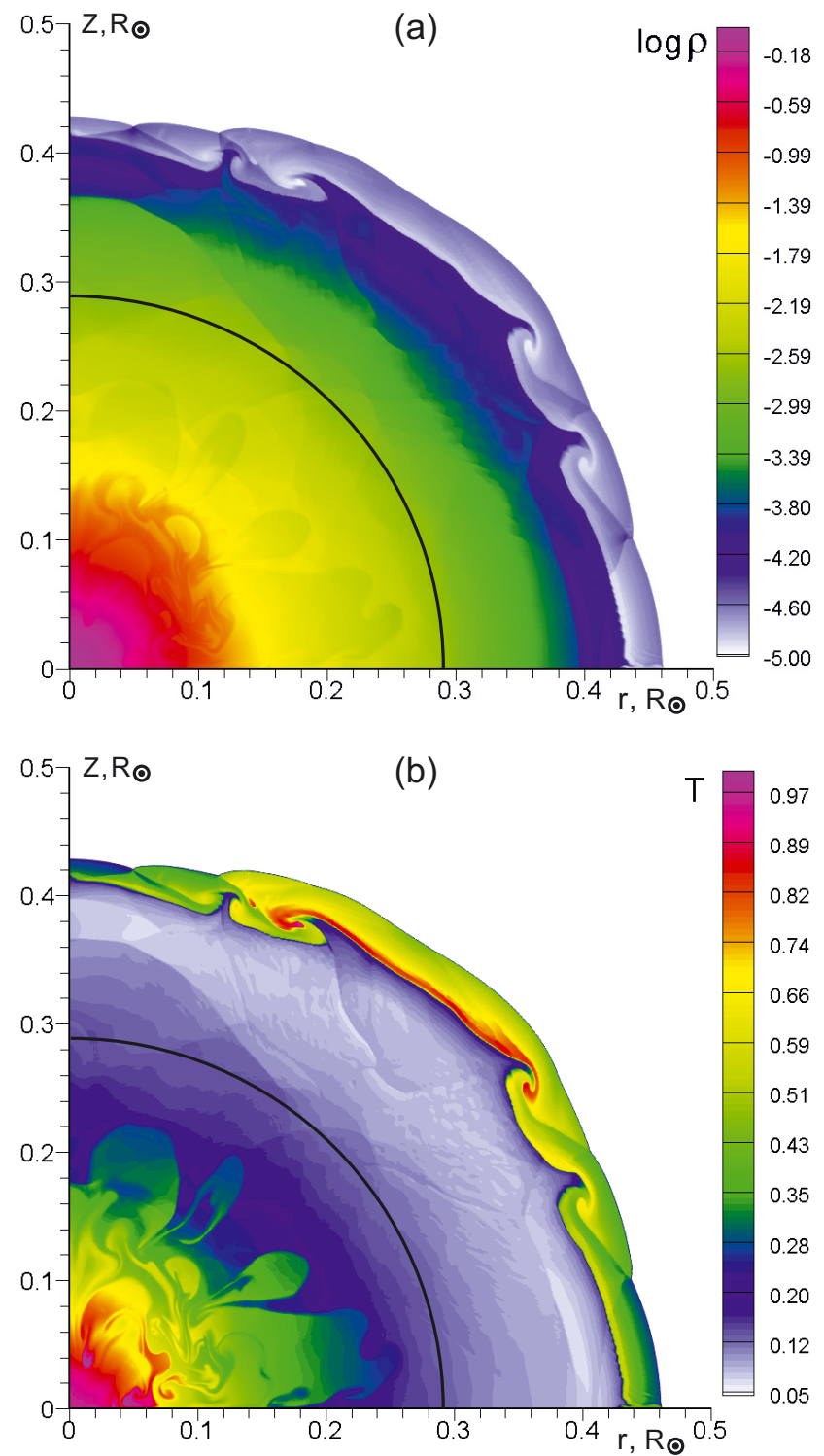

Fig. 4. SN model with multicore ignition for $t=28 \mathrm{~s}$. Logarithm of density a) is shown in units of $\rho_{\mathrm{c}}=2.65 \times 10^{5} \mathrm{~g} / \mathrm{cm}^{3}$. Temperature b) is shown in units of $T_{\mathrm{c}}=2.36 \times 10^{9} \mathrm{~K}$.

produced by the explosion, is split on two fronts that are propagating through the rarefied matter and heating it. In the central part of the core, there is a region with a Rayleigh-Taylor instability. The radius at which this instability occurs is very close to the value obtained in Chen et al. (2011). The development of the instability requires more precise computations with a more physical configuration of the envelope, but we suppose that this instability could result in the formation of large-scale nonuniform structures on a large time scale.

To cure the pathological "carbuncle" behavior that could appear in a region where the shock is almost but not exactly parallel to the grid edges, the artificial dissipation was inserted according to Loh \& Jorgenson (2009). This is the reason for the instability development in Fig. 3 being smoothed near the $z$-axis.

Figure 4 presents the density and temperature for multicore ignition. In this scenario the front of the shockwave is not as smooth as in the previous case. Many fragments of hot matter appear behind the shockwave. This could lead to disrupting of the star in many fragments. As a result the light curves 
of such supernova could be more complex than in a spherically symmetric case.

\section{Discussions and conclusions}

We have presented our analysis of a PISN explosion. Results of a $1 \mathrm{D}$ simulations are in good agreement with previous works. We proposed the initial binding energy of a star as the criteria of its subsequent fate. An interesting correlation between total nuclear energy release and maximum temperature was found. We also performed 2D numerical simulations. We proposed to explore two possible models of PISN explosion mechanism: uniform and nonuniform explosion.

The spherically symmetric explosion in general reproduces the results of other numerical codes (Chen et al. 2011; Joggerst et al. 2011); however depending on how nuclear explosion occurs in massive star, we could imagine different pictures of the stellar explosion. If the stellar core is nonuniform prior to explosion, nuclear burning could start in some parts of the core. To simulate this model we proposed a multicore ignition scenario. This could be an "exotic scenario" and not the standard explored to now. But if the explosion is nonuniform, it could change the light curve, chemical production, and also the spectrum.

Another key question of PISN explosion phenomena is the role of the envelope. Woosley et al. (2007) propose the idea that quite small pulsations of a pair-unstable star could eject the envelope. They applied their results to the case of SN2006gy, which is known to be a PISN. If we apply an idea of nonuniform explosion to a star without an envelope, it could produce a light curve with very complex behavior that is different from the plateau type. But this problem requires additional investigation by radiation-transfer computations.

Acknowledgements. The computations were performed on MVS-100K of the Joint Supercomputer Center of the Russian Academy of Sciences. The authors are grateful to Vladimir Bakhtin (Keldysh Institute of Applied Mathematics) for assistance in the parallel computations by DVM-system software. The work was supported by the Russian Foundation for Basic Research (project nos. 12-0100606a, 12-02-00687a, 12-02-31737mol_a), Basic Research Programs No. 15 and No. 21 of the Presidium of the Russian Academy of Science and by the scientific school NSh-1434.2012.2. Andrey Baranov and Anastasia Filina are supported by the Erasmus Mundus Joint Doctorate Program by Grants Number 2010-1816, 2012-1710 from the agency EACEA of the European Commission.

\section{References}

Almgren, A. S., Beckner, V. E., Bell, J. B., et al. 2010, ApJ, 715, 1221 Arnett, W. D. 1972, ApJ, 176, 699

Arnett, W. D. 1973, Explosive Nucleosynthesis, eds. D. N. Schramm, \& W. D. Arnett (Austin: Univ. of Texas Press), 236

Arnett, W. D. 1974, Late Stages of Stellar Evolution, eds. R. J. Tayler, \& J. E. Hesser (Dordrecht: D. Reidel Publishing), IAUS 66, 1

Arnett, W. D. 1996, Supernovae and Nucleosynthesis (Princeton: Princeton University Press), 380

Arnett, W. D., \& Meakin, C. 2011, ApJ, 733, 78

Ayasli, S., \& Joss, P. S. 1982, ApJ, 256, 637

Barkat, Z., Rakavy, G., \& Sack, N. 1967, Phys. Rev. Lett., 18, 379

Blinnikov, I. S., Dunina-Barkovskaya, N. V., \& Nadyozhin, D. K. 1996, ApJS, 106,171

Bond, J. R., Arnett, W. D., \& Carr, B. J. 1984, ApJ, 280, 825

Bromm, V., \& Larson, R. B. 2004, ARA\&A, 42, 79

Bromm, V., Yoshida, N., \& Hernquist, L. 2003, ApJ, 596, L135

Chardonnet, P., Chechetkin, V., \& Titarchuk, L. 2010, Ap\&SS, 325, 153

Chen, K.-J., Heger, A., \& Almgren, A. S. 2011, Comput. Phys. Commun., 182, 254

Colella, P., \& Woodward, P. R. 1984, J. Comput. Phys., 54, 174

Dai, W., \& Woodward, P. R. 1994, J. Comput. Phys., 115, 485

de Souza, R. S., Yoshida, N., \& Ioka, K. 2011, A\&A, 533, A32

Dunina-Barkovskaya, N. V., \& Imshennik, V. S. 2003, Astron. Lett., 29, 10

Fraley, G. S. 1968, Ap\&SS, 2, 96

Fowler, W. A., \& Hoyle, F. 1964, ApJS, 9, 201

Glatzel, W., El Eid, M. F., \& Fricke, K. J. 1985, A\&A, 149, 413

Heger, A., \& Woosley, S. E. 2002, ApJ, 567, 532

Joggerst, C. C., \& Whalen, D. J. 2011, ApJ, 728, 129

Kasen, D., Woosley, S. E., \& Heger, A. 2011, ApJ, 734, 102

Kritsuk, A. G., Ustyugov, S. D., Norman, M. L., \& Padoan, P. 2009, J. Phys. Conf. Ser., 180, 012020

Loh, C. Y., \& Jorgenson, P. C. E. 2009, J. Comput. Phys., 228, 1343

Nozawa, T., Kozasa, T., Umeda, H., Maeda, K., \& Nomoto, K. 2003, ApJ, 598, 785

Ober, W. W., El Eid, M. F., \& Fricke, K. J. 1983, A\&A, 119, 61

Popov, M. V. 2012, Comput. Mathem. Mathem. Phys., 52, 1186

Popov, M. V., \& Ustyugov, S. D. 2007, Comput. Mathem. Mathem. Phys., 47, 1970

Rakavy, G., \& Shaviv, G. 1967, ApJ, 148, 803

Rakavy, G., \& Shaviv, G. 1968, ApJS, 1, 429

Rakavy, G., Shaviv, G., \& Zinamon, Z. 1967, ApJ, 150, 131

Ustyugov, S. D., Popov, M. V., Kritsuk, A. G., \& Norman, M. L. 2009, J. Comput. Phys., 228, 7614

Waldman, R. 2008, ApJ, 685, 1103

Wheeler, J. C. 1977, Ap\&SS, 50, 125

Woosley, S. E., Heger, A., \& Weaver, T. A. 2002, Rev. Mod. Phys., 74, 1015

Woosley, S. E., Blinnikov, S., \& Heger, A. 2007, Nature, 450, 390 